\title{
EFFECT OF DEHYDRATION ON BLOOD TESTS
}

\section{Case scenario}

A 75 year old lady was admitted with history of cough, high grade fever and reduced oral intake for 4 days. She also complained of reduced urine output. Her past medical history included Type 2 Diabetes, Hypertension, migraine, GORD, and a non-functioning pituitary microadenoma. Her regular medication included Hydrochlorothiazide, Amlodipine, Metformin and Omeprazole. She was found to have dry mucous membranes, temperature $38.2 \mathrm{C}^{\circ}$, and BP 90/60 $\mathrm{mmHg}$. Chest examination revealed $\mathrm{L}$ sided basal crackles. Her blood tests on admission and after starting iv fluid replacement are summarized in table 1.

Table 1

\begin{tabular}{|l|l|l|l|}
\hline Tests & $09 / 03$ & $11 / 03$ & $13 / 03$ \\
\hline $\mathrm{Hb}(\mathrm{g} / \mathrm{L})$ & 135 & 111 & 113 \\
\hline WBC & $24.2 \times 10^{9}$ & $16.9 \times 10^{9}$ & $12 \times 10^{9}$ \\
\hline Haematocrit & 0.443 & 0.368 & 0.369 \\
\hline MCV & 96.9 & 96.1 & 94.4 \\
\hline
\end{tabular}

\begin{tabular}{|ll|l|l|l|}
\hline $\mathrm{Na}^{+}$ & $\mathrm{mmol} / \mathrm{L}$ & 160 & 156 & 150 \\
\hline $\mathrm{K}$ & $\mathrm{mmol} / \mathrm{L}$ & 4.4 & 3.4 & 3.4 \\
\hline Urea & $\mathrm{mmol} / \mathrm{L}$ & 31.3 & 18.9 & 9.9 \\
\hline Creatinine & (umol/L) & 263 & 145 & 133 \\
\hline Glucose & (mmol/L) & 19 & 11 & 9 \\
\hline HbA1c & 75 & & \\
\hline
\end{tabular}

\begin{tabular}{|l|l|l|l|}
\hline Total proteins (g/L) & 60 & 48 \\
\hline Albumin (g/L) & 29 & & 23 \\
\hline ALT (IU/L) & 24 & & 23 \\
\hline ALP (IU/L) & 132 & 126 \\
\hline $\begin{array}{l}\text { Total bilirubin } \\
\text { (umol/L) }\end{array}$ & 08 & 10 \\
\hline Total Globulin (g/L) & 31 & 28 \\
\hline
\end{tabular}

The effect of dehydration on blood tests highlighted in green colour indicates that Haemoglobin, Haematocrit, urea/creatinine, $\mathrm{Na}^{+} / \mathrm{K}^{+}$, Glucose and protein levels change significantly with improvement in hydration status of the patient. A number of questions arising from this data are:

1- Is the poor glycaemic control a marker of dehydration or uncontrolled diabetes?

2- Is drop in haemoglobin due to blood loss?

3- Do the renal function tests suggest dehydration as primary cause of AKI?

4- Does the drop in albumin indicate deterioration in the liver's synthetic function?

\section{INTRODUCTION;}

Dehydration is common in patients presenting to the acute admissions ward. The most common reasons include poor oral intake and fluid loss from: 
- Gastrointestinal tract (eg, diarrhoea, vomiting,)

- Skin (eg, fever, burns)

-Urine (eg, glucosuria, diuretic therapy, diabetes insipidus, DKA)

A reduction of the central circulating blood volume due to hypovolemia accompanying dehydration, results in a fall in cardiac filling pressure and stroke volume and, if uncompensated, a fall in cardiac output. The body can compensate by moving water from the extravascular to the intravascular space. ${ }^{1-2}$ As a result of these fluid shifts, changes in electrolytes and water concentrations in various body compartment occur which are reflected in many blood tests results. This is classically seen in patients with Diabetic Ketoacidosis and Hyperglycaemic Hyperosmolar State (previously HONK).

The clinical and biochemical features of dehydration ${ }^{3}$ are summarized in table 2 .

Table 2

Clinical features of dehydration

Dry mucus membrane

Dry skin

Reduced skin turgor

Reduced axillary sweating

Orthostatic hypotension

Tachycardia and hypotension (indicates shock)

Cognitive impairment

Reduced urinary output [ $<0.5 \mathrm{ml} / \mathrm{kg} / \mathrm{h}$ is suggestive of acute kidney injury (AKI)]

Concentrated urine and high osmolality

\section{Biochemical changes}

Raised serum urea

Raised creatinine

Reduced estimated glomerular filtration rate (eGFR)

Increased urea:creatinine ratio

Hypernatraemia (loss of water greater than salt loss)

Raised serum or urine osmolality

Raised urine specific gravity

\section{Effects of dehydration on Haemoglobin, Hematocrit \& HbA1C}

Both haemoglobin and haematocrit increase in a dehydrated person. ${ }^{2,4}$ Hiroshi Nose ${ }^{1}$ and colleagues induced dehydration in 10 subjects by exercise and checked haemoglobin $(\mathrm{Hb})$, haematocrit (Hct), $\mathrm{Na}, \mathrm{K}^{+}, \mathrm{Cl}$, and plasma osmolality at 0 mins, 30 mins and 60 mins after exercise. Figure 1 shows the change in $\mathrm{Hct}, \mathrm{Hb}$, and plasma solids before and after dehydration. Immediately after exercise, these increased from $42.7 \pm 0.5 \%$ to $44.7 \pm 0.5 \%, 14.8 \pm 0.2 \mathrm{~g} / \mathrm{dl}$ to $15.8 \pm 0.2 \mathrm{~g} / \mathrm{dl}$, and $8.4 \pm 0.1 \mathrm{~g} / \mathrm{dl}$ to 9.1 $\pm 0.1 \mathrm{~g} / \mathrm{dl}$, respectively. The significant differences observed before and after dehydration were maintained for the next $60 \mathrm{~min}$. 

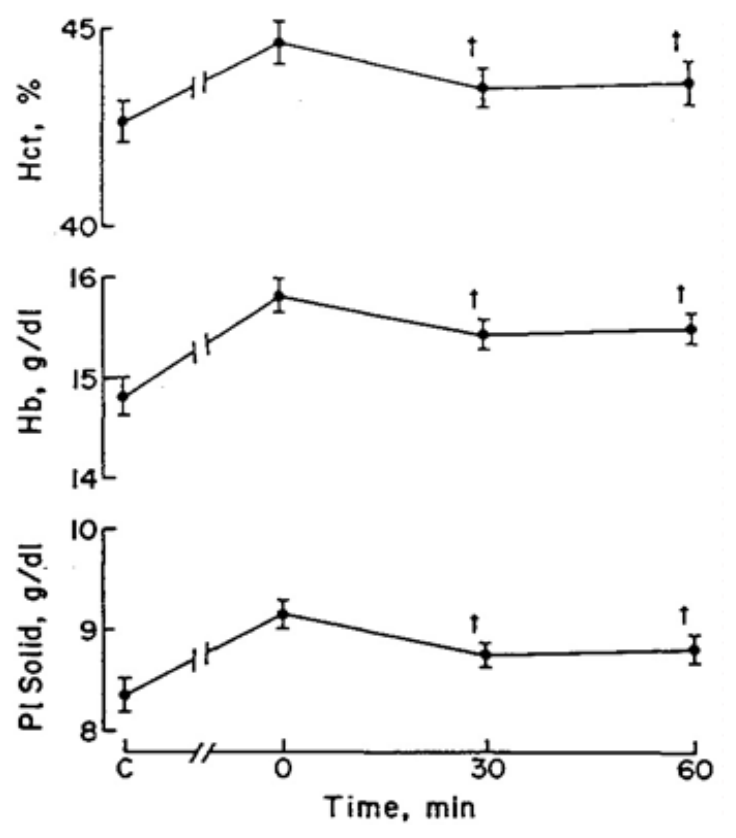

FIGURE 1 Hematocrit (Hct), hemoglobin (Hb) concentration, and plasma (PI) solids are shown as means \pm SE of 10 subjects before $(C)$ and 0,30 , and 60 min after dehydration. Significant differences were observed for all variables between control and dehydrated conditions (0,30, and 60 rain).

Significant differences between 0 min and the other 2 dehydrated conditions (30 and $60 \mathrm{~min}$ ).

$\mathrm{HbA1c}$ (glycated haemoglobin) is the measure of glycaemic status of an individual over last 3 month ${ }^{5}$. It is formed by non-enzymatic reaction which occurs between glucose and the $\mathrm{N}$-end of the beta chain. ${ }^{5}$ There is very little literature available on data search to suggest that dehydration directly affects $\mathrm{HbA1c}$. However a rise in urea level as a result of dehydration can alter the $\mathrm{HbA1c}$ test results depending on the assay. ${ }^{8}$ One study showed that in patients with uraemia, glycated haemoglobin measured by ion exchange chromatography was significantly elevated, but this was not correlated with the degree of glucose intolerance ${ }^{6}$. This was due to the excessive amount of cyanate derived from the urea, which causes carbamylation at the $\mathrm{N}$-terminal valine residue. This carbamylated haemoglobin (carbHb) results in an increase in the $\mathrm{HbA1}(\mathrm{a}+\mathrm{b})$ and hence, the increased levels of HbA1. ${ }^{7,8}$ However, newer ion-exchange HPLC assay methods show improved separation of the $\mathrm{HbA} 1 \mathrm{c}$ fraction from other haemoglobin fractions and therefore no interference from carbHb. ${ }^{9}$

\section{Effect of dehydration on CBG measurements}

Hypotension as a result of dehydration results in decrease in perfusion and increase in glucose utilization in the local tissue leading to false low results of capillary blood glucose tests. One study assessed the validity of the capillary blood glucose measurements in the hypotensive, critically-ill patients. Capillary glucose values were significantly lower than those obtained from testing venous blood on the reagent strips and also lower than laboratory glucose measurements. Capillary glucose values in the hypotensive group were $33 \%$ lower than venous laboratory glucose values and were significantly lower than the values obtained in the normotensive group. ${ }^{10}$

\section{Effect of dehydration on Blood Glucose}

One study has looked at the effect of dehydration in frogs and demonstrated that dehydration can increase blood glucose levels. ${ }^{11}$ The rise in glucose was found to be out of proportion to changes in metabolite concentrations that could be due to passive concentration of the plasma (haemoconcentration) as a result of dehydration. Glucose was significantly elevated even in $12.2 \%$ 
dehydrated frogs and rose progressively to a final level 23.6 times higher than controls in $50 \%$ dehydrated frogs.

Another study showed an increase in hepatic glucose production, with increased plasma glucose levels during hyperosmolality which can be caused by dehydration ${ }^{12}$. The very high levels of venous glucose seen in patients with Hyperglycaemia Hyperosmolar State often resolve rapidly with rehydration alone without the need for insulin. This would suggest a significant effect of dehydration on venous glucose concentration.

\section{Effect of dehydration on Renal Function tests}

Dehydration has multiple effects on the kidney. The loss of body water leads to an increase in serum osmolality and activation of vasopressin which results in urinary concentration ${ }^{13}$. This can be seen clearly in the above given case scenario.

Nose ${ }^{1}$ and colleagues also demonstrated sustained effects on plasma electrolyte concentration before and after dehydration (Figure 2).
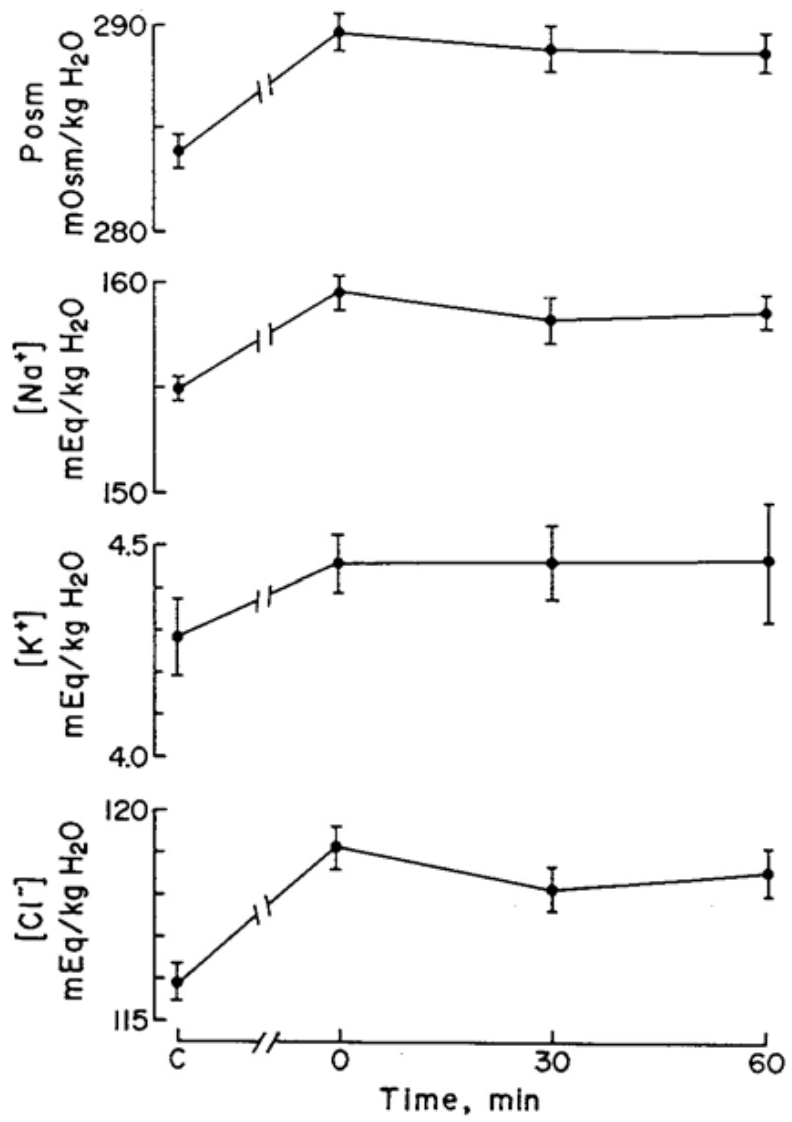

FIGURE 2 Changes in electrolyte concentrations and osmolality (Posmol) in plasma after dehydration. Significant differences were observed for all variables between control and dehydrated conditions $(0$, 30 , and $60 \mathrm{~min}$ ).

However there is an exception in the case of patients with Cranial Diabetes Insipidus (CDI). Dehydrated patients usually present with an elevated serum urea level, owing in part to increased renal reabsorption of urea mediated by antidiuretic hormone (ADH). Serum urea values fall in patients with ADH deficiency (CDI) and this fact can be used to distinguish patients dehydrated 
because of $C D I$ from those with usual hypertonic dehydration and intact $A D H$ secretion. In one study, the mean serum urea level was $2.9 \mathrm{mmol} / \mathrm{L}$ in the $\mathrm{CDI}$ group and $15.4 \mathrm{mmol} / \mathrm{L}$ in the patients without CDI while the mean serum sodium level was $155 \mathrm{mmol} / \mathrm{L}$ in both groups. ${ }^{13}$

\section{Effect of dehydration on lipid profile}

The effect of dehydration on lipid profile has been investigated in fasting subjects. ${ }^{14}$ Subjects were fasted, initially with no fluid replacement and then with salt and water supplementation. Subjects who had fasted with no fluids had a higher total serum cholesterol, HDL cholesterol, LDL cholesterol, apolipoprotein A-1, and apolipoprotein B, compared to subjects who had fasted with prior fluid and salt replacement.

\section{Effect of dehydration on Liver Function Tests}

The above given case scenario demonstrates a significant difference in serum total proteins and albumin levels in a dehydrated patient, before and after hydration with intravenous fluids. However, bilirubin and liver enzymes levels remained unchanged, indicating that changes in protein levels were essentially due to hydration status rather than liver abnormality per se.

\section{Conclusion}

Clinicians should take hydration status of patient into account before interpreting the laboratory results. Before routine blood tests, patients should avoid unnecessary physical activity, avoid hot dry environments, ensure adequate intake of water, and avoid diuretic substances such as caffeine.

\section{$\underline{\text { Key Points }}$}

1- HbA1c values can vary depending on assay, because of rise in urea level as a result of dehydration, however, there is no interference with modern assays.

2- Capillary blood glucose values in a dehydrated person can be significantly lower than the values obtained by venous reagent strips or laboratory glucose measurements.

3- Dehydration can increase the blood glucose levels per se.

4- Dehydration leads to an increase in plasma osmolarity and urea levels, seen particularly in HHS.

\section{References}

1. Medicine, I. of. Fluid Replacement and Heat Stress. Nutrition Research (1994). doi:10.17226/9071

2. Nose, H., Morimoto, T. \& Ogura, K. Distribution of water losses among fluid compartments of tissues under thermal dehydration in the rat. Jpn. J. Physiol. 33, 1019-29 (1983).

3. El-Sharkawy, A. M., Sahota, O., Maughan, R. J. \& Lobo, D. N. The pathophysiology of fluid and electrolyte balance in the older adult surgical patient. Clin. Nutr. 33, 6-13 (2014).

4. Holsworth, R. E., Cho, Y. I. \& Weidman, J. Effect of hydration on whole blood viscosity in firefighters. Alternative Therapies in Health and Medicine 19, 44-49 (2013).

5. Bunn, H. F., Haney, D. N., Kamin, S., Gabbay, K. H. \& Gallop, P. M. The biosynthesis of human 
hemoglobin A1c. Slow glycosylation of hemoglobin in vivo. J. Clin. Invest. 57, 1652-1659 (1976).

6. de Boer, M. J., Miedema, K. \& Casparie, A. F. Glycosylated haemoglobin in renal failure. Diabetologia 18, 437-440 (1980).

7. Standing, S. J. \& Taylor, R. P. Glycated haemoglobin: an assessment of high capacity liquid chromatographic and immunoassay methods. Ann. Clin. Biochem. 29 ( Pt 5), 494-505 (1992).

8. Fluckiger, R., Harmon, W., Meier, W., Loo, S. \& Gabbay, K. H. Hemoglobin carbamylation in uremia. N Engl J Med 304, 823-827 (1981).

9. Little, R. R. et al. MEASUREMENT OF HbA1c IN PATIENTS WITH CHRONIC RENAL FAILURE. Clin Chim Acta 418, 73-76 (2013).

10. Fingerstick Glucose Determination in Shock_Annals of Internal Medicine _American College of Physicians.

11. Churchill, T. A. \& Storey, K. B. Metabolic effects of dehydration on an aquatic frog, Rana pipiens. J. Exp. Biol. 198, 147-154 (1995).

12. Keller, U., Szinnai, G., Bilz, S. \& Berneis, K. Effects of changes in hydration on protein, glucose and lipid metabolism in man: impact on health. Eur. J. Clin. Nutr. 57 Suppl 2, S69-S74 (2003).

13. Comtois, R., Bertrand, S., Beauregard, H. \& Vinay, P. Low serum urea level in dehydrated patients with central diabetes insipidus. Cmaj 139, 965-969 (1988).

14. Campbell NR, Wickert W, Magner P, S. S. Dehydration during fasting increases serum lipids and lipoproteins. Clin Invest Med. 17, 570-6 (1994). 